\title{
El profesor de educación en tecnología y su lucha por mantener su saber disciplinar vigente
}

\section{The technology education professor and his struggle to keep his disciplinary knowledge current.}

\author{
Claudia Esperanza Saavedra Bautista ${ }^{1}$ \\ Luis Alfonso Salcedo Plazas ${ }^{2}$ \\ Sandra Elizabeth Suárez Páez ${ }^{3}$
}

\section{RESUMEN}

Este escrito tiene como propósito examinar los retos que sortean los educadores dedicados a la enseñanza de la educación en tecnología desde la línea de la informática educativa y su lucha por mantener su saber disciplinar vigente, reconociendo que este campo se caracteriza 1 Doctora en ciencias de la educación, Docente de la Licenciatura en Tecnología, Facultad Seccional Duitama, Universidad Pedagógica y Tecnológica de Colombia, Duitama, Colombia, Grupo de investigación RESET, claudia. saavedra@uptc.edu.co, Orcid: https://orcid.org/0000-00027981-4378

2 Magister en Ciencias Matemáticas, Especialista en Matemática y Estadística Aplicada, Docente de la Escuela de Matemáticas y Estadística, Facultad de Ciencias, Universidad Pedagógica y Tecnológica de Colombia, Tunja, Colombia, Grupo de Investigación GAMMA, luis.salcedo@ uptc.edu.co, Orcid: http://orcid.org/0000-0001-6246-014X $3 \quad$ Magister en Administración y Supervisión Educativa, Docente de la Licenciatura en Tecnología. Facultad Seccional Duitama, Universidad Pedagógica y Tecnológica de Colombia, Duitama, Colombia, Grupo de investigación RESET, sandra.suarez@uptc.edu.co, Orcid: https://orcid. org/0000-0002-3877-3506

por su acelerada actualización de herramientas a nivel de hardware y de software obligando al docente a buscar procesos de alfabetización disciplinar constante. El abordaje metodológico se realiza desde el paradigma interpretativo con enfoque cualitativo, aplicando un método histórico documental, que a lo largo del análisis permitió develar los grandes desafíos que han tenido que asumir los educadores en el campo de la informática como expresión de la tecnología, pues una tecnología que hoy se califica como vigente, en poco tiempo termina siendo obsoleta; por lo que esta dinámica ha retado constantemente el actuar del educador en tecnología e informática y la pertinencia del perfil profesional del educador en tecnología. 


\section{PALABRAS CLAVE:}

Educador en Tecnología, Informática educativa, Saber disciplinar, Tecnología e Informática, TIC.

\section{ABSTRACT}

The purpose of this writing is to examine the challenges faced by educators dedicated to teaching technology education from the line of educational informatics and their struggle to maintain their current disciplinary knowledge, recognizing that this field is characterized by its accelerated updating of tools. at the hardware and software level, forcing the teacher to seek constant disciplinary literacy processes. The methodological approach is carried out from the interpretive paradigm with a qualitative approach, applying a documentary historical method, which throughout the analysis allowed to reveal the great challenges that educators in the field of computer science have had to face as an expression of technology, since a technology that today is classified as current, in a short time ends up being obsolete; Therefore, this dynamic has constantly challenged the actions of the technology and information technology educator and the relevance of the professional profile of the technology educator.

\section{KEYWORDS:}

Teaching role, Know how to discipline, Technology and Informatics, ICT.

\section{INTRODUCCIÓN}

La llegada de las TIC a territorios escolares han avizorado diversos retos que abren discusiones en torno a la necesidad de desarrollar competencias TIC en los docentes, a la importancia de dotaciones tecnológicas en las instituciones educativas, las resistencias de los docentes frente al uso e integración de las TIC, entre otros desafíos encaminados a promover mayor participación y democratización del conocimiento en estudiantes y docentes; sin embargo, estas discusiones se han venido dando desde un escenario general, pues poco se ha hablado de los desafíos que enfrenta un docente que se ha formado para orientar en el campo de la educación en tecnología, específicamente en la línea de la informática educativa y cuya presencia de las herramientas TIC le ha sido familiar desde tiempo atrás. En este sentido, los resultados que se señalan en este texto hacen parte de un estudio doctoral más amplio denominado "Integración disciplinar de las TIC en la formación de educadores para el área de Tecnología e Informática en la UPTC. (1994-2015)" que se desarrolló desde un método histórico documental comparado con enfoque interpretativo y que buscaba analizar el devenir histórico de dos programas académicos dedicados a formar educadores en el campo de la educación en tecnología. Sin embargo, en este escrito se privilegia aspectos asociados a los desafíos disciplinares que enfrenta el profesor de la línea de informática educativa en un programa de formación de educadores de educación en Tecnología de una Universidad Colombiana y su lucha por mantener su saber disciplinar vigente.

\section{EL SABER DISCIPLINAR DEL EDUCADOR EN TECNOLOGÍA}

El saber disciplinar del docente se caracteriza por el dominio conceptual y práctico de un campo de conocimiento específico, el cual se distingue de otros saberes como el saber pedagógico, el saber académico y el saber transdisciplinar. Al respecto (Molano et al., 2016) basado en (Ibarra, 2009) plantea que:

"existe una tensión entre la formación disciplinar y la formación pedagógica, dado que la primera considera tener su propia teoría del conocimiento y su didáctica específica, y dice no necesitar formación pedagógica, restringiéndola a la revisión del pensamiento de algunos educadores. Otra perspectiva supone 
que el conocimiento científico o técnico por sí solo no es suficiente en la práctica educativa, siendo necesaria la formación en pedagogía y el reconocimiento de los contextos donde se enseña, las características del estudiante y en la necesidad de privilegiar los procesos sociales emergentes en el aula". (p.9)

En el caso particular del saber disciplinar del profesor de educación en tecnología, específicamente en la línea de informática educativa, se ha observado de manera permanente la necesidad de moldear y reconfigurar constantemente su saber disciplinar, teniendo en cuenta que la llegada de nuevas herramientas y tendencias en este campo ocurre en forma ágil e imprevista, por lo que este educador debe desaprender conceptos y acciones prácticas que se usan a través de lenguajes y programas de software que pasan a ser obsoletas, para aprender aquellas que van llegando en forma imprevista y que se van posicionando en este campo de estudio. En efecto, para el área Informática el perfil de egreso tiene dificultades particulares especiales, pues el origen reciente y la rápida evolución que experimentan la informática y las tecnologías asociadas, hacen que los conocimientos y las técnicas que la sustentan estén en permanente cambio (Contreras, 2013, p. 17).

Sin embargo, a pesar de los retos que su praxis pedagógica embiste, es pertinente reconocer que los educadores del campo de la informática educativa han sido agentes importantes en los procesos de formación y alfabetización tecnológica de educadores en diferentes áreas del conocimiento, que han contribuido a disminuir las brechas digitales que se han generado producto de la llegada de la tecnología, brindando herramientas que permiten integrar las TIC en forma transversal. También, su rol ha aportado a los intereses de políticas públicas nacionales como a la "Ruta de apropiación de TIC en el desarrollo profesión docente" (MEN, 2008) y las "Competencias TIC para el desarrollo profesional docente" (MEN, 2013) pero también a lineamientos internacionales como los "Estándares de competencias en TIC para docentes" (Unesco, 2008), que buscan promover el uso y apropiación de TIC por los docentes de todas las áreas de conocimiento y en los diferentes niveles educativos.

Desde el contexto latinoamericano el perfil para un profesional del área de informática que se plantea (Contreras, 2013) desde el proyecto Tuning para América Latina Tuning América busca responder a las tendencias del futuro para lo cual plantea como Meta-perfil para el área de informática que:

El profesional informático
latinoamericano aporta al
desarrollo de la sociedad y
de las organizaciones donde
participa, con las capacidades
y habilidades que le confieren
sus conocimientos de
computación, de tecnologías de
la información, de sistemas y de
organizaciones, sumadas a una
formación integral, sustentada
en la ética profesional, la
responsabilidad social y el
compromiso con la calidad.
Aplica sus conocimientos con un alto nivel de abstracción, lo que le permite identificar, plantear y resolver problemas, aportando soluciones fundamentadas en las ciencias de la computación y las tecnologías de la información. Asimismo, se distingue por su capacidad para investigar y aprender nuevos enfoques, técnicas y paradigmas de la disciplina, actualizando y ampliando sus conocimientos 
y habilidades prácticas
permanentemente. (p. 48)

Para el caso de los educadores en Tecnología es importante mediar el saber pedagógico con el saber disciplinar, de modo que se configure una identidad disciplinar y pedagógica que responda a las necesidades de un área curricular que está en constante transformación debido a los acelerados cambios de la tecnología. Según Cabero (1999) el perfil de un Tecnólogo Educativo, sugiere: a) Determinar proyectos apropiados para el desarrollo Instruccional; b). Valorar las características de la comunidad educativa; c) Analizar las características estructurales de trabajo tareas y contenidos; d) Comunicar efectivamente en forma visual, oral y escrita. e) Demostrar apropiadas conductas interpersonales de grupo y consultoría. f) Promover la difusión y adopción de procesos de desarrollo tecnológico; g). Identificar la ideología de los medios de información y comunicación; h). Informar de los resultados de su investigación y cómo serán utilizados; i) Mantener la confidencialidad de la información que se recoja de los participantes; j) Ofrecer un conocimiento tecnológico en educación, pertinente, eficiente y eficaz.

\section{METODOLOGÍA}

El desarrollo de esta investigación se asumió desde un enfoque cualitativo, entendido desde Denzin \& Lincoln (1998) como una perspectiva multimetódica, naturalista e interpretativa, donde el investigador asume el reto de indagar contextos naturales para interpretar o dar sentido al fenómeno estudiado. Para abordar el componente cualitativo se implementó la técnica de análisis de contenido que desde los planteamientos de Berelson (1952) se entiende como una técnica de investigación que pretende ser objetiva y sistemática en el estudio del contenido manifiesto de la comunicación.
La configuración metodológica de este estudio se consolida desde un método de investigación histórico documental que, según Ruíz (1976) se despliega en cuatro fases: heurística, crítica, hermenéutica y exposición. La primera fase se orientó a la ubicación, clasificación y sistematización de las fuentes primarias y secundarias; la segunda fase, sobre el análisis crítico de las fuentes, estuvo asociada con la validez y confiablidad de las fuentes; la tercera fase, definida como hermenéutica se ocupó de la interpretación histórica de los datos obtenidos durante las fases iniciales y la cuarta fase, que hace referencia a la exposición, se concentró en la construcción histórica desde el análisis de los documentos revisados y los resultados obtenidos de una encuesta aplicada a 106 graduados del programa objeto de estudio con perfil de profesional de educadores en el campo de la informática educativa.

La aplicación de la encuesta tuvo como propósito recoger percepciones y apreciaciones con el fin de aportar al análisis y contrastar los hallazgos documentales asociados con los retos que debe sortear el educador en tecnología de la línea disciplinar de informática educativa por mantener su saber disciplinar vigente. La encuesta se organizó en 17 preguntas abiertas y cerradas que se analizaron desde los constructos e indicadores de análisis que se sugería en la revisión documental. En la tabla 1. se presentan los constructos que se analizaron. 
Tabla 1.

Constructos de análisis

\begin{tabular}{|c|l|}
\hline Constructo & \multicolumn{1}{|c|}{ Descripción } \\
\hline Datos de contexto & $\begin{array}{l}\text { Permite conocer la titulación, cargo y el año de graduación para describir las } \\
\text { percepciones de los graduados en cada periodo de estudio. }\end{array}$ \\
\hline Percepción del programa & $\begin{array}{l}\text { Indaga sobre la percepción que tienen los graduados sobre el programa con } \\
\text { respecto a la formación recibida y la contribución en el perfil profesional. }\end{array}$ \\
\hline $\begin{array}{c}\text { Saberes disciplinares } \\
\text { predominantes }\end{array}$ & $\begin{array}{l}\text { Examina los saberes disciplinares que predominaron durante la formación } \\
\text { recibida e identifica los vacíos de conocimiento disciplinar que perciben los } \\
\text { graduados. }\end{array}$ \\
\hline Necesidades del contexto & $\begin{array}{l}\text { Busca reconocer las necesidades y amenazas de los graduados en el contexto } \\
\text { laboral. }\end{array}$ \\
\hline laboral & $\begin{array}{l}\text { Evalúa la apreciación de los graduados con respecto a su participación en las } \\
\text { reformas curriculares que ha realizado el programa. }\end{array}$ \\
\hline graduados & $\begin{array}{l}\text { Explora sobre las estrategias de actualización en el campo profesional que } \\
\text { utilizan los graduados. }\end{array}$ \\
\hline Procesos de actualización
\end{tabular}

Nota. Elaboración propia.

Así mismo, con el objeto de validar la confianza del instrumento, se envió a expertos donde surgieron algunos ajustes de estructura $y$ redacción, posteriormente se aplicó a cada una de las preguntas el método Alpha de Cronbach, que consiste en una prueba estadística que valida la confianza, fiabilidad y homogeneidad de las preguntas para determinar las correlaciones que existen entre las mismas, de modo que al utilizar la información recopilada se ofrezcan resultados estables y consistentes. Los resultados de validación del instrumento arrojaron un Alpha de Cronbach superior al 0.80 , considerándose un valor confiable y se manejó un intervalo de confianza del $95 \%$ con un margen de error del $5 \%$.

El análisis de las preguntas abiertas que incluía la encuesta se realizó en el marco de un enfoque cualitativo mediante de una exploración hermenéutica, donde a través de una codificación abierta del contenido recuperado, se fueron tomando las referencias que aportaban a los constructos definidos dentro de la estructura de la encuesta e identificando categorías emergentes. Para ello, se apoyó del software de análisis de datos cualitativos Nvivo. En el caso del análisis estadístico se utilizaron los softwares Excel y SPSS- Statistical Package for the Social Sciences.

Para poder reconocer las preocupaciones disciplinares del educador de la línea de informática educativa, adicional de la encuesta aplicada se revisaron los contenidos programáticos que se orientaron durante el periodo 1994-2015 en el programa académico objeto de estudio y se recurrió al diseño de una matriz basada en los saberes digitales que permitió identificar la evolución del plan de estudio de los programas durante el periodo analizado. Los saberes digitales han sido propuestos por Ramírez, Casillas y Ojeda (2015) y permiten analizar procesos de integración de TIC desde la determinación de los saberes digitales, pues "estar alfabetizado tecnológicamente- no solamente tiene que ver con el manejo de software especializado, sino 
con otros saberes digitales que permitan que el estudiante o profesor universitario pueda realizar tareas propias de su disciplina" (p.135). Por tanto, para este estudio los saberes digitales se concibieron como marcos de referencia para analizar los retos y desafíos del profesor de la línea de informática educativa.

En suma, los saberes digitales hacen referencia saber usar dispositivos, saber administrar archivos, saber usar programas y sistemas de información especializado, saber crear y manipular contenido de texto y texto enriquecido, saber crear y manipular conjuntos de datos, saber crear y manipular medios y multimedia, saber comunicarse en entornos digitales, saber socializar y colaborar en entornos digitales, saber ejercer y respetar una ciudadanía digital, y por último el saber digital asociado con literacidad digital.

\section{DISCUSIÓN Y RESULTADOS}

\section{TENDENCIAS Y DESAFÍOS DISCIPLINARES DEL EDUCADOR EN TECNOLOGÍA}

Para analizar la evolución de las tendencias curriculares asociadas con la integración de las TIC como componente disciplinar en la formación de educadores de los programas objeto de estudio, se tuvo en cuenta dos criterios: de una parte, las asignaturas que se establecieron en el área específica de la informática al momento de creación del programa, y de otra, se tomó como referente las asignaturas que se mantuvieron en el tiempo dentro del plan de estudios, esto, para poder hacer la trazabilidad de los cambios más importantes a nivel curricular.

Es así, que se tomaron en total quince asignaturas las cuales, a pesar de sufrir algunos ajustes nominativos, se mantuvieron en los dos periodos de estudio y que corresponden a: Lógica matemática, Estructuras de programación I, Estructuras de programación II, Estructuras de programación III, Estructuras de programación IV, Utilidades I, Utilidades II, Análisis de sistemas, Bases de datos, Diseño de sistemas, Software educativo I, Software educativo II, Telemática.

La matriz de saberes digitales se utilizó como rejilla para revisar la evolución del conocimiento disciplinar a la luz de los saberes disciplinares, encontrando cambios significativos en tres líneas como son: programación y producción de software educativo, multimedia educativa y sistemas y redes de computadores. En la siguiente tabla se presenta, la evolución desde los saberes digitales en la línea de la informática educativo en el periodo analizado. 
Tabla 2.

Evolución de los contenidos disciplinares de la línea disciplinar de Programación y producción de software educativo desde los saberes digitales.

\begin{tabular}{|c|c|c|}
\hline Saberes Digitales & $\begin{array}{c}\text { Programación y producción de } \\
\text { software educativo } \\
\text { (1994-2004) }\end{array}$ & $\begin{array}{l}\text { Programación y producción de software } \\
\text { educativo } \\
(2005-2015)\end{array}$ \\
\hline $\begin{array}{l}\text { 1. Saber usar } \\
\text { dispositivos }\end{array}$ & $\begin{array}{l}\quad \text { Retroproyector; Video Beam; } \\
\text { Computador; Uso de dispositivos de } \\
\text { almacenamiento secundario. }\end{array}$ & $\begin{array}{l}\text { Retroproyector; Video Beam; } \\
\text { computador; aula virtual; tablero digital }\end{array}$ \\
\hline $\begin{array}{l}\text { 2. Saber administrar } \\
\text { archivos }\end{array}$ & $\begin{array}{l}\text { Diseño de archivos, bases de datos } \\
\text { en Access. }\end{array}$ & $\begin{array}{l}\text { Sistemas de gestión de Bases de datos } \\
\text { en SQL, consultas de información en SQL. }\end{array}$ \\
\hline $\begin{array}{l}\text { 3. Saber usar } \\
\text { programas y sistemas } \\
\text { de información } \\
\text { especializados }\end{array}$ & $\begin{array}{l}\quad \text { Programación estructurada. } \\
\text { Lenguajes de programación: Lenguaje } \\
\text { Logo } \\
\text { Lenguaje Basic; Lenguaje Pascal; } \\
\text { Lenguaje C; Lenguaje PROLOG. } \\
\text { Programas: Turbo C para windows, } \\
\text { Macromedia MX; Linux RedHat 9.0; } \\
\text { Introducción al desarrollo de sistemas } \\
\text { de información } \\
\text { Programación al desarrollo de software } \\
\text { educativo; Etapas y metodológicas para } \\
\text { el desarrollo de software; Desarrollo de } \\
\text { un software educativo. }\end{array}$ & $\begin{array}{l}\text { Programación orientada a objetos. } \\
\text { Lenguajes de programación: Lenguaje } \\
\text { Java, JavaScript, Visual Basic; Action Script } \\
2.0 \text { y } 3.0 \text {; XML; HTML, XHTML; PHP;mysql, } \\
\text { introducción a android; HTML5; CSS3; API } \\
\text { de Canvas. } \\
\text { Programas: Authorware, Tool book } \\
\text { VRML (Lenguaje Modelado de Realidad } \\
\text { Virtual), Dfd, DevC++ o Turbo C 3.1, Rhide o } \\
\text { XWPE para entorno Unix. } \\
\quad \text { Diseño de páginas web, Windows XP, } \\
\text { Plug-in CosmoPlayer, editor } \\
\text { VrmIPad 1.2, Internet Scene Assembler, } \\
\text { Dreamveawer, html, aplicaciones web, } \\
\text { aplicaciones en Macromedia } 8 \text { (Flash, } \\
\text { Dreamweaver y Diorector), Microsoft } \\
\text { Acces; NetBeans: Eclipse; Apache; Xampp }\end{array}$ \\
\hline $\begin{array}{l}\text { 4. Saber crear y } \\
\text { manipular contenido } \\
\text { de texto y texto } \\
\text { enriquecido }\end{array}$ & & \\
\hline
\end{tabular}




\begin{tabular}{|c|c|c|}
\hline Saberes Digitales & $\begin{array}{c}\text { Programación y producción de } \\
\text { software educativo } \\
\text { (1994-2004) }\end{array}$ & $\begin{array}{l}\text { Programación y producción de software } \\
\text { educativo } \\
(2005-2015)\end{array}$ \\
\hline $\begin{array}{l}\text { 5. Saber crear y } \\
\text { manipular conjuntos } \\
\text { de datos }\end{array}$ & $\begin{array}{l}\text { Lógica y Algoritmos; Diagrama } \\
\text { de flujo y pseudocódigo; Tomas de } \\
\text { decisión; Ciclos repetitivos; Vectores } \\
\text { o Arreglos; Tipos de datos; Variables; } \\
\text { Estructuras de un programa; Sentencias } \\
\text { de asignación; Manejo de cadenas } \\
\text { de caracteres; Procedimientos } \\
\text { funciones; Registros; Archivos; Pasos } \\
\text { de parámetros a funciones; Listas; } \\
\text { Matrices dispersas; Colas y pilas; } \\
\text { Sistemas de información } \\
\text { organizacionales; ciclo de vida de } \\
\text { un sistema; herramientas para } \\
\text { determinar requerimientos del sistema; } \\
\text { estrategias de desarrollo por análisis } \\
\text { estructurado; estrategias de desarrollo } \\
\text { por prototipo; transición de análisis a } \\
\text { el diseño; diseño de salidas del sistema } \\
\text { de cómputo; diseño de entrada y } \\
\text { controles diseño del diálogo en línea. } \\
\text { Introducción a bases de datos; } \\
\text { Sistema de gestión de bases de } \\
\text { datos; bases de datos en Access. } \\
\text { Diagramación uML }\end{array}$ & $\begin{array}{l}\text { Algoritmos, diagramas de flujo; } \\
\text { metodologías de programación, } \\
\text { Programación orientada a objetos, } \\
\text { Vectores, Matrices, Apuntadores, } \\
\text { recursividad Herencia simple, Herencia } \\
\text { múltiple, Polimorfismo; manejo de bases } \\
\text { de datos; sistemas de gestión de Bases de } \\
\text { datos en SQL, consultas de información } \\
\text { en SQL; Conceptos Básicos: Bases de } \\
\text { Datos; Independencia, Arquitectura de un } \\
\text { Sistema de Bases de Datos; Pasos para el } \\
\text { Diseño e Implementación de una Base de } \\
\text { Datos; esquema lógico y modelo de datos; } \\
\text { El modelo E/R; Diseño de bases de datos } \\
\text { relacionales por descomposición; Ciclo de } \\
\text { vida de una aplicación informática; Ejercicios } \\
\text { de aplicación UM; sistemas numéricos. } \\
\text { Servlets; Arquitectura Del Paquete } \\
\text { Servlet; Creación de un Servlet; } \\
\text { Interacción con Servlets: Ciclo de vida } \\
\text { de un Servlet; Persistencia de datos. } \\
\text { applets; la etiqueta applets; parámetros; } \\
\text { manejo del navegador desde el applet; } \\
\text { Controles gráficos con applets. }\end{array}$ \\
\hline
\end{tabular}




\begin{tabular}{|c|c|c|}
\hline Saberes Digitales & $\begin{array}{c}\text { Programación y producción de } \\
\text { software educativo } \\
\text { (1994-2004) }\end{array}$ & $\begin{array}{l}\text { Programación y producción de software } \\
\text { educativo } \\
(2005-2015)\end{array}$ \\
\hline $\begin{array}{l}\text { 6. Saber crear y } \\
\text { manipular medios y } \\
\text { multimedia }\end{array}$ & 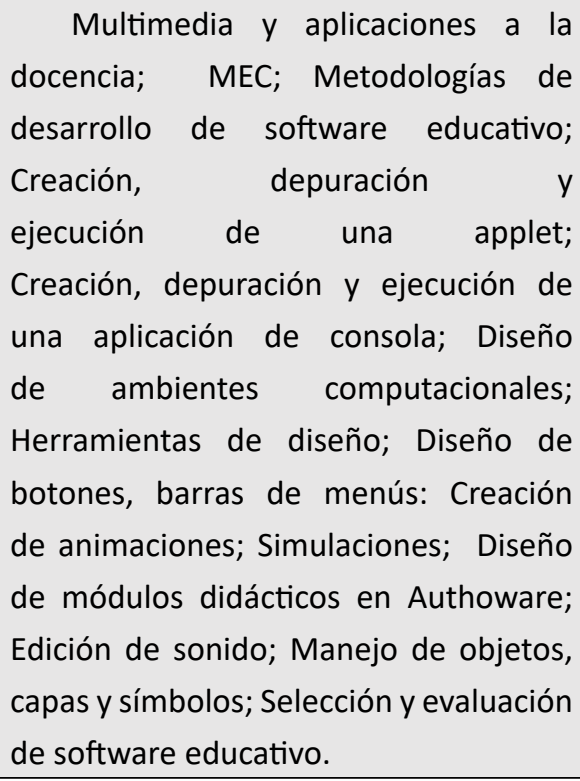 & $\begin{array}{l}\text { Uso de Authorware para la creación } \\
\text { de software educativo; desarrollo de } \\
\text { aplicaciones en Toolbook; Comandos, } \\
\text { funciones y arreglos en Toolbook; } \\
\text { creación de ambiente de aprendizaje } \\
\text { computarizados; creación de aplicaciones } \\
\text { para la web; Diseño de páginas web: } \\
\text { Creación de aplicaciones en JAVA ambiente } \\
\text { grafico (GUI); creación de aplicaciones } \\
\text { móviles para Android, patrones de diseño. }\end{array}$ \\
\hline \multicolumn{3}{|l|}{$\begin{array}{l}\text { 7. Saber comunicarse } \\
\text { en entornos digitales }\end{array}$} \\
\hline \multicolumn{3}{|l|}{$\begin{array}{l}\text { 8. Saber socializar y } \\
\text { colaborar en entornos } \\
\text { digitales }\end{array}$} \\
\hline $\begin{array}{l}\text { 9. Saber ejercer } \\
\text { y respetar una } \\
\text { ciudadanía digital }\end{array}$ & & \\
\hline 10. Literacidad digital & & \\
\hline
\end{tabular}

Nota. Elaboración propia basada en la revisión de los contenidos programáticos de las asignaturas.

La mirada histórica a la luz de los saberes digitales mostró que no en todas las líneas disciplinares (programación y producción de software educativo; multimedia educativa; sistemas y redes de computadores) se ubicaron los saberes digitales; sin embargo, hubo saberes digitales que se hicieron presentes en más de una línea disciplinar. En el caso de la línea de programación y producción de software educativo, no se hicieron presentes los saberes digitales: Saber crear y manipular contenido de texto y texto enriquecido; Saber comunicarse en entornos digitales; Saber socializar y colaborar en entornos digitales; Saber ejercer y respetar una ciudadanía digital; Literacidad digital.
Sin embargo, en este periodo se empezó a utilizar el uso de la plataforma virtual Moodle, que seguramente contribuyó al desarrollo de los saberes digitales asociados con el saber comunicarse en entornos digitales.

No obstante, se observan fortalezas en los saberes digitales asociados con saber usar dispositivos; saber administrar archivos; saber usar programas y sistemas de información especializados; saber crear y manipular conjuntos de datos; saber crear y manipular medios y multimedia. Pues se observó que desde sus inicios el programa ha tenido una predominancia en el componente de 
programación de computadores y el desarrollo de software educativo.

De otra parte, dentro del periodo en estudio se evidenció que el software especializado más representativo que se utilizó para el desarrollo educativo y contenidos multimediales fueron ToolBook, Authorware y Flash con apoyo de software de diseño gráfico como Corel Draw, Photoshop y Fireworks. Así mismo, se hizo evidente desde la línea de programación, la transición de pasar de la programación estructurada en el primer periodo de estudio a implementar en el segundo periodo la enseñanza de una programación orientada a objetos, donde para el primer periodo se implementaron lenguajes de programación como: Lenguaje Logo, Basic; Pascal; PROLOG y C, mientras que en el segundo periodo, emergieron lenguajes y aplicativos basados en: Java, JavaScript, Visual Basic; Action Script 2.0 y 3.0 ; XML; HTML,
XHTML; PHP; mysql, Android; HTML5; CSS3; API de Canvas.

A nivel de discurso, en cuanto a la producción de software, se hablaba de "ambientes computacionales", mientras en el segundo periodo, la referencia estaba un poco más enfocada al contexto educativo, pues se planteaba el diseño de "ambientes virtuales de aprendizaje" "aplicaciones móviles educativas" pensando en los propósitos pedagógicos que se pretendían alcanzar a través de la interacción del estudiante con el material diseñado.

Ahora bien, revisando la segunda línea disciplinar que se identificó como tendencia curricular desde el componente de la "informática educativa", aparece la "multimedia educativa", para lo cual producto de la revisión histórica a los contenidos programático se establecieron las siguientes tendencias a la luz de los saberes digitales.

Tabla 3.

Evolución de la línea disciplinar de Multimedia educativa desde los saberes digitales

\begin{tabular}{|l|l|l|}
\hline \multicolumn{1}{|c|}{ Saberes Digitales } & \multicolumn{1}{|c|}{$\begin{array}{c}\text { Multimedia educativa } \\
\text { (1994-2004) }\end{array}$} & $\begin{array}{c}\text { Multimedia educativa } \\
\text { (2005-2015) }\end{array}$ \\
\hline 1. Saber usar dispositivos & $\begin{array}{l}\text { Retroproyector; Video Beam; } \\
\text { Sistema de cómputo; Unidad central } \\
\text { de procesos partes } \\
\text { Dispositivos de entrada y salida de } \\
\text { datos; Unidad de almacenamiento } \\
\text { secundario; Introducción a los } \\
\text { computadores; Videos } \\
\text { Cámara filmadora; Cámara } \\
\text { fotográfica; Casetes; medios de } \\
\text { almacenamiento. }\end{array}$ & $\begin{array}{l}\text { Computador, medios de } \\
\text { almacenamiento, Video beam, proyector } \\
\text { digital, }\end{array}$ \\
\hline $\begin{array}{c}\text { 2. Saber administrar } \\
\text { archivos }\end{array}$ & \multicolumn{1}{|c|}{$\begin{array}{c}\text { Manejo de formatos } \\
\text { dabletas, tablero }\end{array}$} \\
\hline
\end{tabular}




\begin{tabular}{|c|c|c|}
\hline Saberes Digitales & $\begin{array}{l}\text { Multimedia educativa } \\
\text { (1994-2004) }\end{array}$ & $\begin{array}{l}\text { Multimedia educativa } \\
\qquad(2005-2015)\end{array}$ \\
\hline $\begin{array}{l}\text { 3. Saber usar programas } \\
\text { y sistemas de información } \\
\text { especializados }\end{array}$ & $\begin{array}{l}\text { Programas: derive; Harvard } \\
\text { graphics; ToolBook, Photoshop; } \\
\text { CorelDraw; Macromedia Flash MX; } \\
\text { Paquetes informáticos como apoyo } \\
\text { a la docencia; Sistemas operativos; } \\
\text { interacción con el ambiente } \\
\text { Windows XP; Antivirus. }\end{array}$ & $\begin{array}{l}\text { Photoshop, CorelDraw, clic, } \\
\text { Windows, Flash 8; paint; Power Point; } \\
\text { Fireworks; Hojas de estilo CSS; K-Sketch. }\end{array}$ \\
\hline $\begin{array}{l}\text { 4. Saber crear y manipular } \\
\text { contenido de texto y texto } \\
\text { enriquecido }\end{array}$ & $\begin{array}{l}\quad \text { Ofimática; Internet; Manejo de } \\
\text { herramientas informáticas básicas; } \\
\text { Procesamiento de datos; Procesador } \\
\text { de palabra; La hoja electrónica y las } \\
\text { matemáticas; Asistente de funciones } \\
\text { en excel; } \\
\text { hipertextos; Presentaciones de } \\
\text { Información; Conceptos básicos } \\
\text { de internet; Combinación de } \\
\text { correspondencia; Manejo de } \\
\text { hipervínculos con documentos } \\
\text { internos y externos; Manejo de } \\
\text { botones de acción; Hipervínculos } \\
\text { StarOffice con Internet; } \\
\text { Programas: Star Office; Open Office. }\end{array}$ & $\begin{array}{l}\text { Ofimática avanzada, editores } \\
\text { gráficos y de Texto, Procesador de } \\
\text { Texto, Hoja de Cálculo, Presentador } \\
\text { de Ideas, Navegador Web; creación y } \\
\text { edición de presentaciones; procesador } \\
\text { de Texto - Microsoft Word Hoja de } \\
\text { Cálculo - Microsoft Excel; Creación de } \\
\text { publicaciones y material de marketing - } \\
\text { Microsoft Publisher }\end{array}$ \\
\hline $\begin{array}{l}\text { 5. Saber crear y manipular } \\
\text { conjuntos de datos }\end{array}$ & $\begin{array}{l}\text { Motores de búsqueda; } \\
\text { Representación interna de datos } \\
\text { Elaboración de gráficas } \\
\text { Bases de datos } \\
\text { Manejos estadísticos } \\
\text { Arquitectura de los sistemas de } \\
\text { bases de datos (Enfoque relacional, } \\
\text { Enfoque jerárquico, Enfoque en } \\
\text { redes) } \\
\text { Manejador de bases de datos (dbase, } \\
\text { foxpro, clipper) }\end{array}$ & \\
\hline
\end{tabular}




\begin{tabular}{|c|c|c|}
\hline Saberes Digitales & $\begin{array}{c}\text { Multimedia educativa } \\
(1994-2004)\end{array}$ & $\begin{array}{l}\text { Multimedia educativa } \\
\qquad(2005-2015)\end{array}$ \\
\hline $\begin{array}{l}\text { 6. Saber crear y } \\
\text { manipular medios y } \\
\text { multimedia }\end{array}$ & $\begin{array}{l}\text { Tutores inteligentes; Edición } \\
\text { de videos; Tutoriales multimedia; } \\
\text { Cartillas multimedia; Creación de } \\
\text { páginas web; Creación de gráficos; } \\
\text { Manejos de formatos; Utilidades } \\
\text { de la multimedia en el proceso de } \\
\text { aprendizaje; Manejo de textos; } \\
\text { Manejo de sonidos; animaciones }\end{array}$ & $\begin{array}{l}\text { Diseño de proyectos } \\
\text { multimediales. Diseño de páginas web, } \\
\text { creación de imágenes; Diseño gráfico; } \\
\text { técnicas de dibujo; computarizadas, } \\
\text { Diseño de MEC's; revistas Enter, Pe } \\
\text { magazín, libros multimediales; diseño } \\
\text { de materiales educativos interactivos; } \\
\text { aplicaciones multimediales en la } \\
\text { educación; Diseño de software } \\
\text { educativo; objetos para la web; } \\
\text { creación de animaciones; ceación y } \\
\text { edición de sonido y video; Aplicaciones } \\
\text { orientadas a procesos educativos }\end{array}$ \\
\hline $\begin{array}{l}\text { 7. Saber comunicarse en } \\
\text { entornos digitales }\end{array}$ & $\begin{array}{l}\text { Introducción a las } \\
\text { comunicaciones } \\
\text { Creación y manejo del correo } \\
\text { electrónico }\end{array}$ & Uso del aula virtual \\
\hline $\begin{array}{l}\text { 8. Saber socializar y } \\
\text { colaborar en entornos } \\
\text { digitales }\end{array}$ & $\begin{array}{l}\text { Introducción a las } \\
\text { comunicaciones } \\
\text { Creación y manejo del correo } \\
\text { electrónico }\end{array}$ & Uso del aula virtual \\
\hline \multicolumn{3}{|l|}{$\begin{array}{l}\text { 9. Saber ejercer y } \\
\text { respetar una ciudadanía } \\
\text { digital }\end{array}$} \\
\hline 10. Literacidad digital & & \\
\hline
\end{tabular}

Nota. Elaboración propia basada en la revisión de los contenidos programáticos de las asignaturas.

En el caso de la línea disciplinar asociada con la "multimedia educativa" se presentó un gran número de saberes digitales, pues en esencia la multimedia en el programa ha permitido promover el diseño creativo de recursos educativo digitales, la alfabetización en el uso de software utilitario como paquetes para ofimática, diseño gráfico, diseño de páginas web, herramientas básicas de la informática, exploración de información especializada en diferentes motores de búsqueda, manejo de formatos de imagen, video, audio y administración de archivos y carpetas, entre otros conocimientos asociados a los saberes digitales de referencia.
Sin embargo, desde el componente disciplinar se observó una ausencia en saberes digitales asociados con saber socializar y colaborar en entornos digitales, saber ejercer y respetar una ciudadanía digital y Literacidad digital, los cuales se desarrollan en el programa, pero desde un componente interdisciplinar.

Durante el primer periodo se hizo evidente que el software de mayor uso fue Star Office; Open Office, Derive, Harvard graphics, ToolBook, Photoshop, CorelDraw y Macromedia Flash MX. Sin embargo, en el segundo periodo, la mayoría de este software se mantuvo en versiones más emergentes y se agregaron otros paquetes como Clic, Dreamweaver, Flash 8; Paint; Fireworks; 
Hojas de estilo CSS; K-Sketch.

En este sentido, se pudo establecer que la multimedia educativa ha sido un área que ha complementado las pretensiones de la línea de programación y producción de software educativo, pues desde ella se van acondicionados saberes que se requieren a la hora de diseñar y desarrollar software con fines educativos. Cabe anotar que, "el software educativo obedece a programas para ordenadores que tienen como objetivo coadyuvar al proceso de enseñanza aprendizaje como un medio didáctico que facilite el proceso. Hay que señalar que su uso se extiende en los años 90 debido al crecimiento generalizado de internet”. (Marquès, 2005)

De otra parte, se observó que la línea disciplinar donde menor presencia hicieron los saberes digitales fue en "sistemas y redes computadores", seguramente porque su tendencia es más técnica y a la luz de los saberes digitales está característica no fue tan visible.

Tabla 4.

Evolución de la línea disciplinar de Sistemas y redes de computadores desde los saberes digitales.

\begin{tabular}{|l|l|c|}
\hline \multicolumn{1}{|c|}{ Saberes Digitales } & \multicolumn{1}{|c|}{$\begin{array}{c}\text { Sistemas y Redes de Computadores } \\
\text { (1994-2004) }\end{array}$} & $\begin{array}{c}\text { Sistemas y redes de computadores } \\
\text { (2005-2015) }\end{array}$ \\
\hline $\begin{array}{l}\text { 1. Saber usar } \\
\text { dispositivos }\end{array}$ & $\begin{array}{l}\text { Retroproyector; Video Beam; } \\
\text { Sistema de comunicaciones; } \\
\text { Medios físicos de transmisión; Cableado } \\
\text { estructurado; Cable coaxial } \\
\text { Cable par trenzado (RJ-45) (directo, } \\
\text { cruzado); Comparación entre hub y } \\
\text { switch. }\end{array}$ & $\begin{array}{l}\text { Retroproyector; Video Beam; } \\
\text { hardware de un computador. }\end{array}$ \\
\hline $\begin{array}{l}\text { 2. Saber administrar } \\
\text { archivos }\end{array}$ & \multicolumn{2}{|l}{} \\
\hline
\end{tabular}




\begin{tabular}{|c|c|c|}
\hline Saberes Digitales & $\begin{array}{l}\text { Sistemas y Redes de Computadores } \\
\text { (1994-2004) }\end{array}$ & $\begin{array}{l}\text { Sistemas y redes de computadores } \\
\qquad(2005-2015)\end{array}$ \\
\hline $\begin{array}{l}\text { 3. Saber usar } \\
\text { programas y sistemas } \\
\text { de información } \\
\text { especializados }\end{array}$ & $\begin{array}{l}\text { Conceptos y definiciones básicas; - } \\
\text { Detección y corrección de errores; } \\
\text { Disciplinas de comunicaciones; Redes de } \\
\text { procesamiento de datos; Redes locales; } \\
\text { Redes públicas; Redes privadas; Servicios } \\
\text { telemáticos; Introducción a las redes } \\
\text { Clasificación de redes según su tamaño: } \\
\text { LAN, MAN y WAN } \\
\text { Clasificación según su distribución lógica; } \\
\text { Conmutación de circuitos, de mensajes y } \\
\text { de paquetes; Comunicación simplex, half- } \\
\text { duplex y full-duplex; Modelo de referencia } \\
\text { OSI. Comparación con el modelo de } \\
\text { Internet; Dominios de colisión; ¿Qué } \\
\text { instalar hubs o switches?; Interconexión } \\
\text { de hubs; Protocolos de la capa de acceso } \\
\text { al medio; Token Ring; Ethernet; Protocolos } \\
\text { de las capas de red y transporte; IPX/SPX; } \\
\text { AppleTalk; Tipos de redes; Topologias } \\
\text { Protocolos de redes LAN; Seguridad en } \\
\text { las redes LAN; RDSI; Frame Relay;STM; } \\
\text { Inteligencia artificial }\end{array}$ & 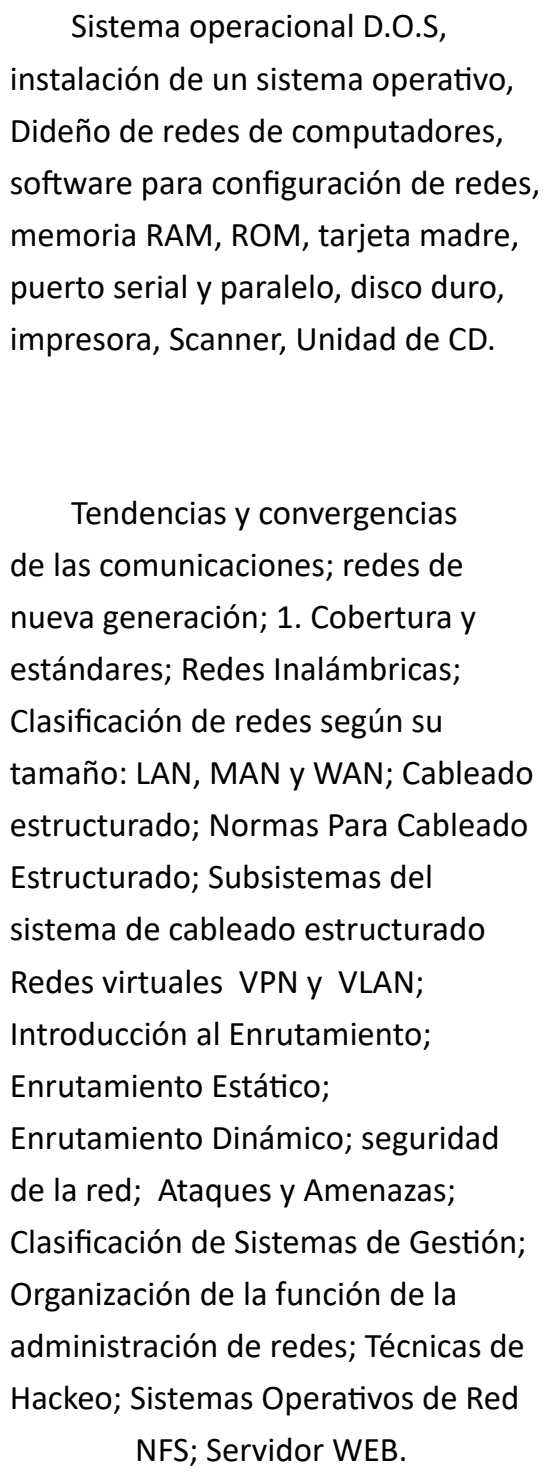 \\
\hline $\begin{array}{l}\text { 4. Saber crear y } \\
\text { manipular contenido } \\
\text { de texto y texto } \\
\text { enriquecido }\end{array}$ & & \\
\hline $\begin{array}{l}\text { 5. Saber crear y } \\
\text { manipular conjuntos } \\
\text { de datos }\end{array}$ & & \\
\hline $\begin{array}{l}\text { 6. Saber crear y } \\
\text { manipular medios y } \\
\text { multimedia }\end{array}$ & & \\
\hline $\begin{array}{l}\text { 7. Saber comunicarse } \\
\text { en entornos digitales }\end{array}$ & & \\
\hline
\end{tabular}




\begin{tabular}{|l|r|r|}
\hline Saberes Digitales & $\begin{array}{c}\text { Sistemas y Redes de Computadores } \\
\text { (1994-2004) }\end{array}$ & $\begin{array}{c}\text { Sistemas y redes de computadores } \\
\text { (2005-2015) }\end{array}$ \\
\hline $\begin{array}{l}\text { 8. Saber socializar y } \\
\text { colaborar en entornos } \\
\text { digitales }\end{array}$ & & \\
\hline $\begin{array}{l}\text { 9. Saber ejercer } \quad \text { respetar una } \\
\text { y } \quad\end{array}$ & & \\
ciudadanía digital & & \\
\hline 10. Literacidad digital & & \\
\hline
\end{tabular}

Nota. Elaboración propia basada en la revisión de los contenidos programáticos de las asignaturas.

A partir de lo anterior, muchas de las temáticas con las que inició el programa se han mantenido en el tiempo, lo que ha cambiado son las herramientas de software y los lenguajes que han emergido a lo largo de la historia, donde el programa en el marco de sus reformas curriculares ha integrado con miras de ofrecer una formación disciplinar actualizada y en sintonía con los acelerados cambios de la tecnología.

De otra parte, se hizo evidente la estrecha relación que guarda la línea de "multimedia educativa" y "programación y producción de software educativo" en cuanto al uso de software y producción de recursos educativos multimediales, pues desde las complejidades de cada línea, en su gran mayoría se enfocó hacia la producción de material educativo multimedial y software educativo, donde uno de los referentes teóricos que con mayor relevancia se ubicó fue Álvaro Galvis Panqueva, quien ha trabajado en cuanto a metodologías de diseño y desarrollo de software educativo, este autor se consolidó como una fuente teórica importante en estas áreas durante el primer periodo y comienzos del segundo periodo.

También, se pudo evidenciar que hasta el año 2015 un programa informático para la producción de software educativo que prevaleció en el tiempo de estudio fue Macromedia Flash MX, que evolucionó a las versiones Flash 8, Adobe flash cs $3, \operatorname{cs} 5$ y cs6; sin embargo, en la actualidad el desarrollo de software educativo se soporta desde otras plataformas más emergentes. Además, se hizo visible que en los comienzos del programa el desarrollo de software educativo se pensó de forma general, sin embargo, en el segundo periodo ya se pensó desde los niveles escolares.

Sin duda la dominancia de saberes digitales es una forma de acondicionar nuevas formar de aprender y enseñar en el marco de una nueva cultura digital, donde el intercambio de conocimiento y la formación de capital tecnológico pueden mejorar a través del acceso a la información, el aprovechamiento de recursos, el enriquecimiento intelectual, la formación académica y la colaboración entre instituciones nacionales e internacionales. (Vázquez, 2009)

\section{EL PERFIL PROFESIONAL DEL EDUCADOR EN TECNOLOGÍA}

El perfil profesional entendido como la promesa disciplinar que persigue un programa académico, se concibe desde Howes (2010) como la declaración formal que hace la institución frente a la sociedad y frente a sí misma, comprometiendo la formación de una identidad profesional, ciertos compromisos formativos en ámbitos de realización de la profesión y sus competencias clave asociadas. Por tanto, el actuar disciplinar del graduado refleja las acciones académicas, investigativas y administrativas que se han hecho dentro del programa académico para consolidar una 
impronta que expresa los saberes específicos que configuran el campo de acción del graduado. Es así que, "la competencia profesional es el grado de utilización de los conocimientos, las habilidades y el buen juicio asociados con la profesión en todas las situaciones que se pueden enfrentar en el ejercicio de la práctica profesional”. (Gómez-Rojas, 2015, p.49)

En este sentido, los programas académicos deben asegurar que las promesas que hacen desde su perfil profesional, se cumplan, pues la realidad es que, un profesional que finaliza sus estudios, sale a enfrentarse a una sociedad compleja, diversa y cambiante, por lo que espera que los saberes que ha forjado durante su paso por el programa académico, se consoliden en una praxis contextualizada que trascienda y derive en una transformación social. Por tanto, se sugiere "pensar el perfil profesional tanto desde los "conocimientos y habilidades como actitudes. Todo esto en conjunto, definido operacionalmente, delimita un ejercicio profesional". (Gómez-Rojas, 2015, p.21). Sin embargo, se ha observado que el perfil profesional en ocasiones absorbe políticas internacionales (Möller,2014), que buscan homogenizar los perfiles y saberes disciplinares, así que, se requiere de un perfil contextualizado que dialogue con los escenarios globales y que no homogenice, pero que mire hacia el mismo horizonte profesional.

Al analizar los perfiles docentes en el programa objeto de estudio, se pudo observar que es común la presencia de graduados del programa que han hecho parte de la planta docente y que han apoyado la enseñanza del componente disciplinar del programa. Por tanto, podría pensarse que la misma fortaleza disciplinar que se ha promovido en el estudiante que se forma en el programa, es la que sale a replicarse una vez ejerce como docente. De otra parte, se observó que, los perfiles docentes que han transitado en el periodo analizado hacen referencia a:
Licenciado en informática educativa, Licenciado en tecnología, Licenciado en tecnología e informática, Licenciado en Educación Industrial, Ingeniero de sistemas e Ingeniero electrónico,

A la luz de esta revisión, se puede observar que a lo largo de la historia, los programas han surtido diferentes procesos de autoevaluación con miras a la acreditación de alta calidad, por lo que los procesos que han enfrentado ante el Consejo Nacional de Acreditación, han permitido revalidar su pertinencia educativa y social, pues la tecnología como campo de naturaleza cambiante, requiere el desarrollo de propuestas curriculares que se sintonicen con las necesidades de la sociedad en el tiempo presente, por lo que la autoevaluación en los programas académicos se ha entendido como una oportunidad para reflexionar críticamente sobre su actuar, de modo de direccionar pertinentemente su función misional.

De igual manera, es común encontrar retos asociados conausenciasanivel de infraestructura en laboratorios o aulas especializadas, por lo que hay un interés constante por fortalecer los laboratorios o lugares de práctica necesarios para el desarrollo de contenidos que se soportan en la experimentación. Al respecto, se puede pensar, que los avances tecnológicos han retado la infraestructura de los programas académicos, pues en la medida que avanzan las tendencias tecnológicas, se deben adquirir nuevos equipos y materiales para el desarrollo de prácticas disciplinares, pues estas se constituyen como experiencias valiosas en la formación, ya que permiten validar conceptos teóricos a través de la experimentación.

Fundamentalmente, conviene señalar que, los programas también han dado relevancia al impacto que tiene sus graduados en el medio, donde producto de la formación recibida han alcanzado diferentes posiciones laborales, entre ellas, docentes en instituciones de educación básica, media y superior, orientadores en 
procesos de alfabetización tecnológica a través de programas nacionales como "Computadores para educar", productores de contenidos audiovisuales educativos, gestores de proyectos asociados con TIC, administradores de infraestructura informática, entre otras posibilidades laborales.

Sin duda, las opciones laborales han sido diversas y se han constituido en un aporte en el desarrollo del país, pues a través de los graduados, los programas responden a su compromiso educativo y social desde el campo de la educación en tecnología e identifican las necesidades y demandas del sector. Por tanto, desde allí se valida y reflexiona la pertinencia curricular del programa y la cohesión social del perfil profesional con el contexto, pues los graduados como un grupo particular dentro de la sociedad, contribuyen a la comprensión de la percepción y el sentido que tiene su actividad profesional y son quienes alertan sobre las necesidades y tendencias que van emergiendo en su campo disciplinar.

Ahora, revisar las perspectivas de los graduados frente al perfil profesional permitió reconocer tendencias disciplinares, problemáticas, necesidades y vacíos de conocimiento que con el paso del tiempo se han agudizado, por tanto, este espacio de reflexión reveló el sentir de los graduados a la luz de su perfil profesional, pues, para los programas debe ser una necesidad imperante el conocer la situación del perfil profesional para alcanzar mayor pertinencia entre la formación que se ofrece y lo que el mercado laboral convoca, de ahí que se requiere una sinergia entre docentes, estudiantes y graduados quienes de manera articulada con las necesidades del contexto sitúen el perfil profesional que se requiere y que espera la sociedad en este campo disciplinar. "La sociedad actual tecnológica, mediática, intercultural, plural, diversa y multifacética, caracterizada por la inmediatez y la comunicación a gran escala pone en jaque a la educación escolar haciendo tambalear sus cimientos en lo que a su finalidad y formas se refiere". (González \& Serrate, 2019, p.61)

De otra parte, los hallazgos obtenidos permitieron reconocer que una forma como los educadores en tecnología luchan por mantener en vigencia su saber disciplinar es a través de procesos de autoformación, participación en eventos académicos y adelantando estudios de posgrado, pues las tendencias que van llegando los motivan a estar en constante actualización de su saber disciplinar. En la siguiente figura se pueden evidenciar las respuestas obtenidas. 
Fig. 1. Estrategias de actualización en el campo profesional Licenciatura en Informática Educativa.

Elaboración propia basada en la encuesta aplicada a graduados.

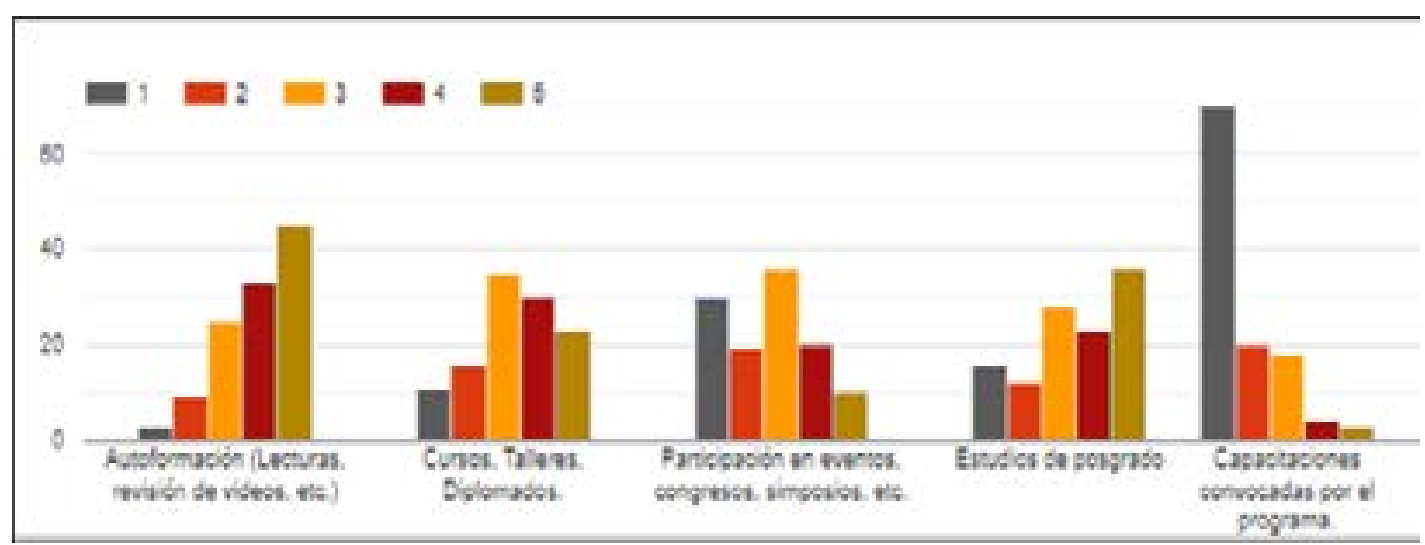

Nota. Elaboración propia.

\section{AMENAZAS AL PERFIL PROFESIONAL DE EDUCADOR EN TECNOLOGÍA}

El análisis realizado a las preguntas abiertas de la encuesta refleja que el señalamiento que se presentó con mayor predominancia fue la fuerte competencia que los ingenieros de sistemas provocan en los concursos docentes, pues su formación técnica se vuelve el "sustituto perfecto" 4 para orientar el área en Tecnología e Informática, desconociendo la importancia que tiene el componente pedagógico para este fin.

También se señaló con fuerte insistencia la falta de actualización en el campo disciplinar, teniendo en cuenta que la tecnología avanza a pasos agigantados y por tanto se requiere de procesos de autoformación constante.

Una amenaza recurrente es el lidiar con contenidos de informática en los cuales no se profundizó durante el pregrado (lagunas disciplinares), lo cual obliga a que como licenciado tenga que autoformarme para suplir estos vacíos, otra amenaza es ver cómo otras áreas como la ingeniería que evidentemente carecen

$\overline{4}$ Tomado de encuesta aplicada a graduados. de formación pedagógica, sustituyan al licenciados que han sido formados para tal labor y cuentan con el perfil profesional adecuado. ${ }^{5}$

De otra parte, es preocupante el valor que se le ha otorgado al área de Tecnología e Informática en las instituciones, pues en algunas, esta asignatura, termina siendo orientada por un docente de cualquier área para completar su carga académica.

Los graduados señalaron la importancia de fortalecer en la formación disciplinar en áreas como electricidad, electrónica, robótica, programación, mantenimiento de equipo de cómputo y redes de computadores, pues en los concursos docentes, los ingenieros de sistemas, eléctricos y electrónicos son quienes con mayor predominancia dominan estos temas, dejándolos por fuera de una convocatoria. Al respecto señalaron que, "Otros profesionales en ingeniería de sistemas, ingeniería electrónica, entre otros, que tienen mayor dominio del área disciplinar sin importar el componente pedagógico que muchas veces adquieren con la experiencia". ${ }^{6}$

\footnotetext{
5 Tomado de encuesta aplicada a graduados.

6 Tomado de encuesta aplicada a graduados.
} 
Entre tanto, la competencia laboral fue otro señalamiento que quedó en evidencia desde el sentir de los graduados, pues, la poca relevancia que se le ha dado al área en algunas instituciones educativa, aunado al alto índice de capital humano para asumirla, ha generado limitadas posibilidades laborales en el gremio de Licenciados en Informática Educativa que se ha preparado para ocuparse de la orientación del área.

\section{CONCLUSIÓN}

El oficio del educador en Tecnología, específicamente en el campo de la informática educativa ha conducido a enfrentar la complejidad de un contexto afectado por el vertiginoso uso de diferentes tecnologías en todos los campos de acción humana y social y ha significado la posibilidad de aportar a la educación desde un área que espera una escuela renovada y con pertinencia social, por lo que a través de los graduados, los programas responden a su compromiso educativo y social desde el campo de la educación en tecnología e identifican las necesidades y demandas del sector. Por tanto, desde allí se valida y reflexiona la pertinencia curricular del programa y la cohesión social del perfil profesional con el contexto, pues los graduados como un grupo particular dentro de la sociedad, contribuyen a la comprensión de la percepción y el sentido que tiene su actividad profesional y son quienes alertan sobre las necesidades y tendencias que van emergiendo en su campo disciplinar.

\section{REFERENCIAS BIBLIOGRÁFICAS}

Contreras, L. (2013). Tuning América Latina. Educación superior en América Latina: reflexiones y perspectivas en Informática.

Cabero Almenara, J. (1999). Tecnología educativa: diversas formas de definirla. Editorial Síntesis, Madrid España.
Berelson B. (1952). Content analysis in communication research. Glencoe III, Free Press.

Denzin, N. \& Lincoln, Y. (1998). Major paradigms and perspectives. Strategies of Qualitative Inquiry, NYK Denzin and YS Lincoln, (eds.) Sage Publication, Thousand Oaks.

González S, \& Serrate, S. (2019). La aportación de los educadores sociales a la secundaria: competencias, funciones y criterios para un nuevo ámbito de trabajo profesional. Educación Social. Revista de Intervención Socioeducativa. Vol. 71 (21), pp. 60-78.

Hawes, G. (2010). Glosario básico para la modernización curricular. Santiago de Chile: Universidad de Chile, Facultad de Medicina, Departamento de Educación en Ciencias de la Salud. Manuscrito no publicado.

Ibarra, O. A. (2009). Saber pedagógico y saber disciplinar ¿Convergencia o Divergencia?

Gómez-Rojas, J. P. (2015). Las competencias profesionales. Revista mexicana de anestesiología, 38(1), 49-55.

Marquès, P. (2005). El software educativo. (Consultado el 11 de abril de 2006). Disponible en: http://rapanui.ucv.cl.

Möller, I., \& Gómez, H. (2014). Coherencia entre perfiles de egreso e instrumentos de evaluación en carreras de educación básica en Chile. Calidad en la Educación, (41), 17-49.

MEN. (2008). Ruta de Apropiación de TIC en el Desarrollo Profesional Docente (pp. 1-20).

MEN. (2013). Competencias TIC Para el Desarrollo Profesional Docente. In Colección Sistema Nacional de Innovación Educativa con uso de Nuevas Tecnologías. https://doi.org/10.1017/ CBO9781107415324.004 
Molano, M., Vargas, W., \& Montealegre, J. (2016). El saber disciplinar escolar en la autobiografía de tres maestros. Journal of Chemical Information and Modeling, 53(9), 1689-1699.

Organización de las Naciones Unidas para la Educación, la C. y la C. (2008). Estándares de Competencia en TIC para docentes. UNESCO, 1-28. http://www.eduteka.org/ pdfdir/UNESCOEstandaresDocentes. pdf\%5Cnhttp://www.eduteka.org/ EstandaresDocentesUnesco.php

Ramírez-Martinell, A., Alvarado, M. A. C., \& Asturias, C. C. C. (2015). La incorporación de las TIC a la enseñanza universitaria de los idiomas. Debate universitario, 3(5), 125-140.

Ruiz, J. (1976). El método histórico en la investigación. Historia de la educación. REP-XXXIV, (134).

Vázquez del Mercado, M. B. (2009). Globalización y educación superior en México. Reencuentro (México, DF), (54), 83-90. 